\title{
Chapter 2: 'Effing Consciousness' - Or, How to Use New Symbols to Get Qualia into the Hard Sciences
}

In the previous Chapter, I started a claim that consciousness science should have a pivotal role in the twenty-first century. Not just in science, but also in society, economy, and the very future of humanity ${ }^{1}$. As part of the early development of that claim, I touched on various kinds of 'consciousness' that science could in principle study. And I pointed to conscious experience as the crucial focus (at least, to start with). I suggested that properly addressing conscious experience will lead to a new incarnation of science - science 3.0. I also suggested that this momentous leap forward must take place, if science simply applies its own logic/experiment requirements more completely and more precisely. Notably, there is 'One Experiment' that will change science forever. Given the rate at which society and economy are evolving, getting consciousness science right is an urgent matter. But as I started to discuss in the last Chapter, the application of scientific rigor to consciousness, and the execution of the One Experiment, are currently blocked by a fragmented and confused field. Of course, fragmentation and confusion are not new to science. We could point to many past periods where science has gone through similar phases, on the way to later understanding. Where we are is not hopeless. But we do need sustained, engaged forward movement, in what is predominantly viewed as a rather esoteric frontier of scientific enquiry. We need to bring consciousness science in from the cold - and set it alight!

The current fragmented state of the field makes forward movement difficult. It's not enough to simply point out the right way to proceed. (And the One Experiment is the right way - as I hope you'll be able to see for yourself.) Some gatekeepers can't currently see the way forward for consciousness science, even when it's presented to them. So, we need to work to develop a common seeing. (This book is part of that effort.) Other gatekeepers don't even agree that consciousness can be studied, scientifically. Thus, there's even more to do than pointing out a scientific path: we need to justify the use of 'scientific' as an adjective! (Again, this book addresses this challenge.) A third group believe that current mainstream approaches are scientific, when they demonstrably aren't! Obviously, there's significant work to be done there, too. (And yes ... this book addresses that issue, too!)

It will take about three-quarters of the book to explain the response I'm suggesting to this tangled situation, in any detail. In the next Chapter, I'll outline the various factions and viewpoints in and around consciousness science. With that overview in hand, I'll be able to sketch a strategic response, designed to bring us all to the One Experiment - and beyond. Chapters 4 to 9 then explain the scientific content of that strategic sketch, at an introductory level. As the last Chapter implied, this scientific explanation isn 't a journalistic report of what's currently going on in consciousness science. It explains a cutting edge that's being resisted by the scientific mainstream. Again, this book is part of a larger body of work that aims to inform non-specialists, in a way that enables them to make up their own minds. Time's too short, and the stakes are too large, to leave these matters just to the specialists. Notably, specialists in narrow scientific disciplines are not necessarily specialists in what humanity needs. Nor are they necessarily experts on the strategic location of science-as-a-whole with respect to those needs. (Chapters 10 through 12 revisit my claims that upgrading consciousness science $i s$ urgent, and significant. By this point, we'll have more of the scientific content in hand. So, we'll be able to get a better sense of the truly pivotal role of consciousness science.) 
In this Chapter, I'm going to do at least three things at once! First, I'm going to illustrate how new scientific content can bridge a current division in the study of consciousness. This shows how we can hope to use logic-and-theory to build a consensus for doing the One Experiment. (This use of logicand-theory is a basic motif in the strategic response discussed and explained in Chapters 3 to 9.) Second, I'm going to show you a new and mostly-unknown scientific advance! Naturally, 'illustrating how new scientific content can bridge divisions' requires explanation of new scientific content. So, of course I'm going to show a scientific advance. But the new content I'm going to show you here is extraordinarily significant: it's a defining building-block for the next phase of science (what I sometimes call 'science 3.0'). (And it will be absolutely pivotal in explaining the One Experiment.) Third, I'm going to introduce you to the subtle and fundamental point about the study of consciousness that's been mostly overlooked, to date. It's a point that we'll keep returning to, because it's intimately related to the One Experiment. So, don't worry if you don't 'get it', straight away. However, if you do - you'll already be on the leading edge of consciousness science. And that also puts you at the leading edge of science $3.0 \ldots$ and makes you an active part, already, of helping humanity get what it needs.

\subsection{The Division: Ineffable vs Scientifically-Appropriate Consciousness}

In the next Chapter, I'll describe a number of different viewpoints about the study of consciousness. Our goal over subsequent Chapters will then be to show how to condense divisions into just two competing positions. Finally, we can construct an experiment that arbitrates between these two alternatives (Figure 2.1A). In this Chapter, I'll provide a preliminary illustration of 'condensing' by addressing just one division, namely what I call the 'ineffability' division.

'Ineffable' means 'unspeakable', or 'beyond description'. The alleged ineffability of conscious experience is one objection to there even being a science of consciousness. After all, if we can't even speak about something, we can't do science on it. In response, I'll show how we can 'eff' conscious experience - i.e. get around the ineffability objection! - by using a new class of symbols. (As I'll go on to show in later Chapters, these new symbols have other vital applications, too.)

For most objections to the path I'm going to describe in this book, there are many variants. That certainly applies to 'speakability' objections. (In fact, a different kind of speakability issue will be at the heart of the One Experiment! ${ }^{2}$ ) In this introductory book, I won't be able to handle every variant of every objection. That's why this book is just an entry-point into a larger body of work (www.theScienceWeNeed.com, Figure 2.1B), including a more-detailed scientific treatment [in particular see Consciousness Science: A New Approach (Rosseinsky, 2019)]. At the end of each Chapter, I give pointers to places you can find discussions of neglected points.

The specific variant of the ineffability objection that I address in this Chapter is well explained by the British philosopher Philip Goff ${ }^{3}$. He claims that science will never explain conscious experience, because modern science is inherently built to handle only things that can be measured in numerical terms, like mass, velocity, and pressure. In contrast, the elements of conscious experience - things like redness of a colour, or the pitch and intensity of a musical note - are not quantifiable. We can easily assign a number to the velocity of a car. What number should we assign to redness, or middle-C-ness? If your immediate response is 'the wavelength of red light' for redness, or 'the frequency of middle C' for middle-C-ness, note that Goff's point relates to the quality of the experience. Consider your firstperson experience of redness as opposed to blueness or greenness. The qualitative, subjective, experiential, difference between redness and blueness is not the same as the quantitative difference between red wavelengths and blue wavelengths. How could we get those subjective differences into science? (Try explaining the difference between redness and blueness to your nearest and dearest! And 
Figure 2.1: Towards the One Experiment - Condensing and Distilling Viewpoints. A: Two Steps Forward. A major problem in the study of consciousness is fragmentation: there are many competing viewpoints, sometimes of a radically-different stance, outlook, or frame. This book is part of a larger body of work that creates two main responses to the split-field issue. Response-1: Use reason and logic (purple arrow) to condense and distill a large number of viewpoints into just two competing issues (green and red rectangles). Note that this process doesn't necessarily mean resolving all the differences in opinion between all the different views. In fact, it primarily means grouping theories together, according to their stance on just one point: does experience couple in a meaningful way with brainactivity, so that information about experience can make its way into verbal and behavioral reports of experience (Figure 2.5). Response-2: Define and conduct an experiment - 'the One Experiment' - to arbitrate between which of the two distilled/condensed viewpoints applies to our inhabited Universe. As we'll see in Figure 2.6, this Experiment then also resolves the most basic issue about consciousness science, which must apply prior to any of the 'multiple theories/views': can we have a science of consciousness? B: The Larger Body-of-Work. The multiple viewpoints from panel A are now arranged at the periphery of two concentric blue-circles, which represent two major structural components of the larger body-of-work (www.theScienceWeNeed.com). The outer circle depicts three books: this one, Reclaiming Reality (Rosseinsky, 2020), and Consciousness Science: A New Approach (Rosseinsky, 2019). These three texts are relatively standalone, integrated, treatments that are aimed at different readerships and different levels. They are inter-consistent, all representing a single viewpoint. And they all draw on a common technical core of about a dozen technical papers, represented by red-stars in the inner blue-circle.

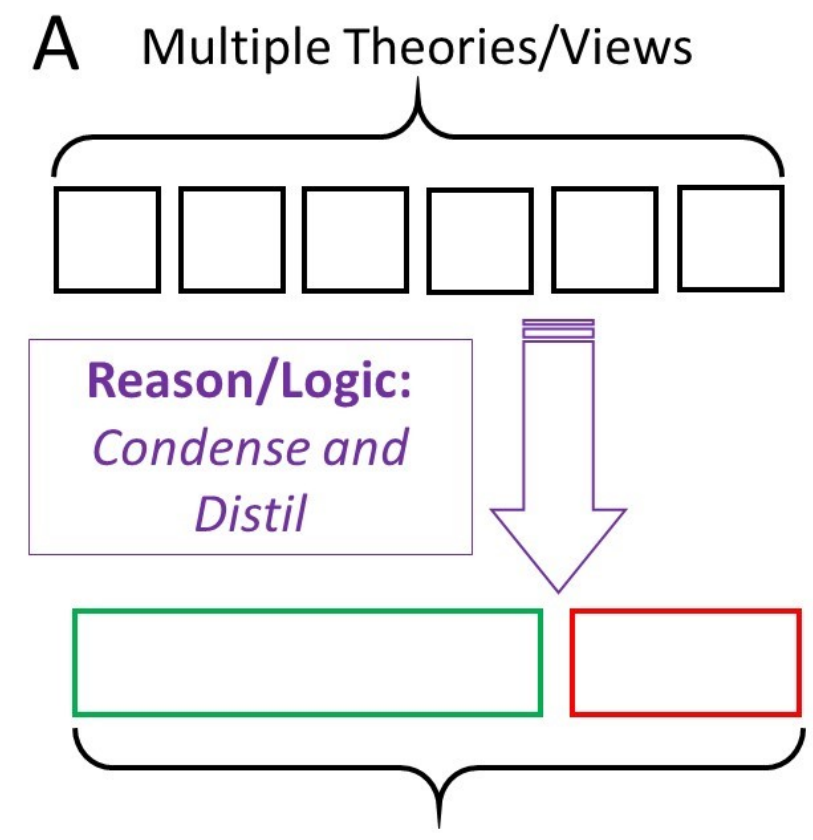

Experimental Test

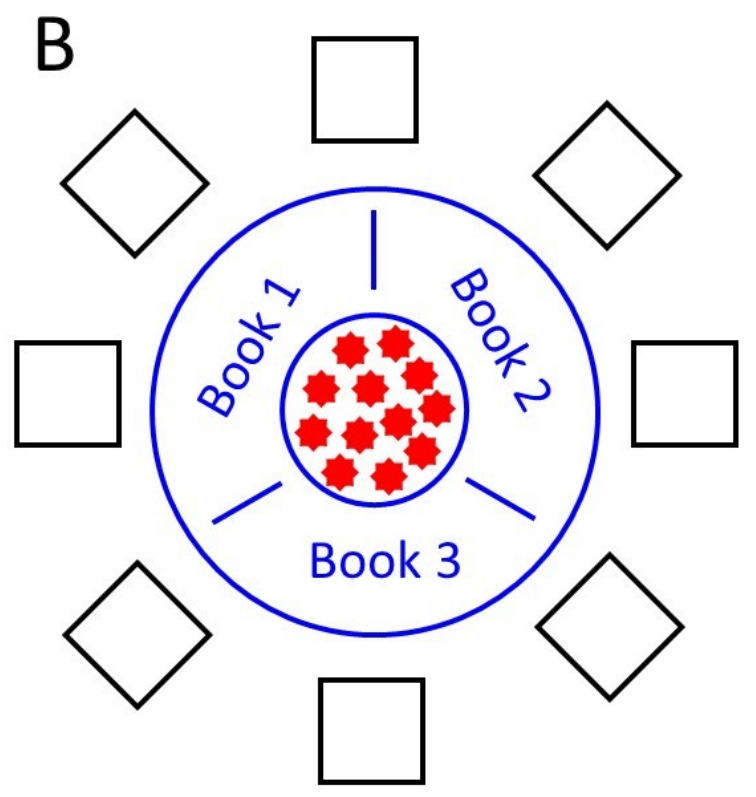

: Technical Paper 
Figure 1.5 (from Chapter 1): One Meaning of 'Consciousness' A. This panel just reproduces Figure 1.1A, adding the red-arrows labelling components of 'conscious experience' (per D1). That is, conscious experience comprises thoughts and feelings (bodily, tactile and emotional), and audiovisual experience of the surroundings. This panel explicitly emphasizes that there is an experienced image or representation of the tree (depicted in colour) and the tree-in-itself (depicted as the purple linedrawing). The 'tree-in-itself' is the collection of atoms/molecules (and, ultimately, quarks, electrons, gluons and photons) that's studied in modern physics. (Defining 'things-in-themselves' in relation to physics gets us out of philosophical abstractions, and into science ... which is where we need to be!) B. This panel shows a different way that people often use the word 'consciousness' - to label just thoughts and feelings, leaving out sights and sounds, which are then taken to 'be' external reality. Words are words, and we can use them in many ways - there's nothing wrong with this usage, e.g. in any absolute sense. However, when we take sights-and-sounds to be objective reality, we lose the contrast between experiential and objective (clearly present in panel A). That means we lose contact with science (because, as Figures 1.3 and 1.4 showed, it's hard to do science under 'direct realism' the proposal that experiential reality is objective reality). And, more importantly, we lose contact with the science/post-Truth conflict. If experiential reality were the same as objective reality (as everyday usage often assumes), there'd be no vulnerability to post-Truth attacks based on subjectivity-inscience. But experiential and objective are not the same: we need to use a panel-A way of looking at things, to first investigate, and then fix, the apparent problems for science which not-the-same creates.
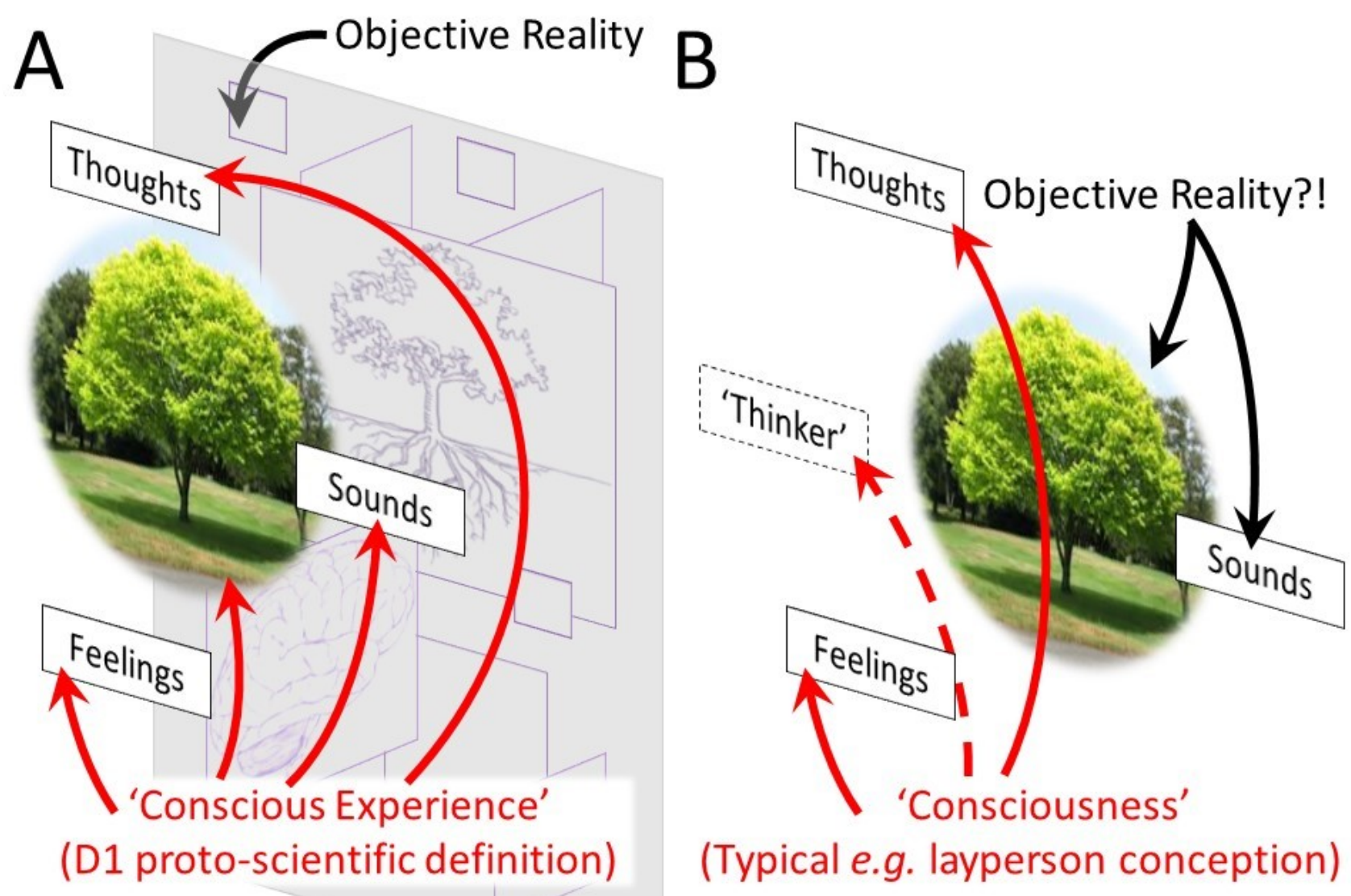

(Typical e.g. layperson conception) 
have them do the same, with you.) If we can't get qualitative subjective differences into science, how could we ever hope to construct a science of experience? (To label this kind of unspeakability as distinct from other kinds, I'll call it 'qualitative-ineffability'.)

\subsection{New Theoretical Content That 'Effs' Conscious Experience}

If you thought of using the numeric wavelength of red light to make 'redness' quantifiable, you were actually on the right track. But the numeric wavelength only stands for the 'red' part. How do get the 'ness' part into science? If the distinction between 'red light' and 'redness' escapes you, look again at Figure 1.5B from Chapter 1. 'Red light' actually exists in what I called 'the unseen realm'. 'Redness' is part of the 'experiential realm'. So, the wavelength description labels a feature in the unseen realm. The qualitative-ineffability problem is that there's something else to label, in the experiential realm (Figure 2.2).

In physics, we usually use the symbol $\lambda$ to label wavelength, and we might introduce a symbol like $\lambda_{\text {red }}$ to label red wavelengths of light. Now, many people have trouble keeping track of scientific symbols (and are sometimes even intimidated by them!). Instead of conventional scientific symbols, in this book I'll generally use italicized words, such as red, to label unseen-realm states. The distinction between unseen-realm and experiential-realm labels will be clear ${ }^{4}$, because right now I'll introduce a new symbol for redness: $\langle r e d\rangle$ ! That is, by putting angle-brackets around a label for an unseen-realm quantity, we get a symbol for the corresponding experiential-realm quality. The 'meaning' of anglebrackets is roughly 'the experience that I have when the thing inside the brackets is present in my sensory environment'. When red-wavelength light, labelled by red, is present, I have an experienceof-redness, labelled by $\langle r e d\rangle$.

Of course, the angle-brackets symbolism only works for unseen-realm symbols that can be directly experienced. A single hydrogen atom can't be directly experienced, so $\langle$ hydrogen atom $\rangle$ is meaningless. There are a lot of other clarifications and nuances required for this angle-bracket symbolism, some of which we'll add as we progress ${ }^{5}$. But for now, we define:

D2: Angle-brackets symbolism. If $s$ is a symbol or label in orthodox science for an unseenrealm 'stimulus' (a feature of reality that can be represented in experience), then $\langle s\rangle$ (pronounced 'angle-brackets-s') is a symbol or label for experience-of- $s$.

As another example, say that a musician is playing the note middle- $C$, in your hearing. The note itself is of course a particular kind of compression wave in the air - it's an unseen-realm thing. We can use angle-brackets symbolism to distinguish between the compression waves and your subjective, qualitative experience of the note: $\langle$ middle-C〉! (Now we have a symbolic answer to the age-old question of 'a tree falling if no one's there to hear it'. We say that compression-waves-in-the-air-dueto-tree-falling happens, whether or not there's a witness. But $<$ compression-waves-in-the-air-due-totree-falling $\rangle$ - or, 'the experienced-sound of a tree falling' - only exists if there's a conscious observer who can convert compression waves into qualitative experience! Of course, we don't need anglebrackets symbolism to get to that understanding - I'm just demonstrating usage.)

Other experiential features of the external environment - for example, the temperature and touch/feel of objects - are straightforward to incorporate into angle-brackets symbolism. Thinking and emotionalfeeling ${ }^{7}$ are a little more complicated, because we don't have a direct external, unseen-realm correlate to put inside the angle-brackets. Seeing-redness and hearing middle-C-ness are direct correlates of red and middle-C. But 'thinking' - as a sort of inner unspoken voice - doesn't necessarily have a direct 
Figure 2.2: New Symbols (Contingently!) Get Subjective Experience into 'Hard Science'. In both panels, the left hand-side of the solid black-vertical line depicts what I'm calling 'phenomena': the unseen-realm above the horizontal dotted-line, and the corresponding experiential-realm below. (The experiencer's brain is omitted here.) To the right of the vertical-line, I create another space, to depict symbols for phenomena. (This space might be thought of as 'existing in' the minds of scientists, so that it's ultimately not separate from 'the world of phenomena', depicted to the left.) This Figure examines the existence of scientific symbols for the unseen-realm (above the dotted line) and for the experientialrealm (below the dotted line). A: As-is science. All symbols in current science properly refer to elements or aspects of the unseen realm. This observation applies at all levels - from symbols for fundamental particles to those for bioproteins or even labels for galaxies! Contrastingly, below the line there are no symbols for subjective, qualitative, experiential features (i.e. no symbols for any aspect of our experientially-lived reality!). B: Symbols to Get 'Qualia' into Hard Science. In a two-step, contingent, process, we first select a special subset of above-the-dotted-line symbols for unseen-reality. These are symbols that label what we might call experienceable features. For example, a symbol red for red-wavelength light. (The Figure uses ' $s_{i}$ ' to stand for a list $s_{1}, s_{2}, \ldots$ etc. of possible stimuluslabels - red, blue, vertical-edge, middle- $C$, and so on.) We then innovate in symbol-space by creating and defining an 'angle bracket' symbolism: $\langle$ red $\rangle$ means experience-of-redness typically induced by sensory contact with unseen-realm red. (In the Figure, the two schematically-depicted tree-edges in the upper-half correspond directly to the brown-green edge experiences in the lower-half. Thus, if the upper-half features were labeled $s_{1}$ and $s_{2}$, the lower-half experiences would be labeled $\left\langle s_{1}\right\rangle$ and $\left\langle s_{2}\right\rangle$.) A tight-coupling of lower-and-upper, concretely grounded in existing upper-definitions, provides a basis for integrating the study of subjective experience (lower-half, experiential realm and symbols) with the hard-science study of objective or unseen reality (upper-half). Nevertheless, I describe the introduction of these symbols as a contingent basis, because they're not enough by themselves (Figure 2.6, Chapter 4, and the One Experiment). We also need to establish an experimental methodology for testing hypotheses stated in angle-bracket symbolism. We'll need angle-bracket symbols themselves to investigate this methodology. In a curious, reflexive, turn, investigation of methodology using anglebracket symbols will then lead us to query whether the statement and definition of those very symbols was meaningful, in the first case! The joint question of existence-of-experimental-methodology and validity-and-meaning-of-symbols will be resolved by the One Experiment!
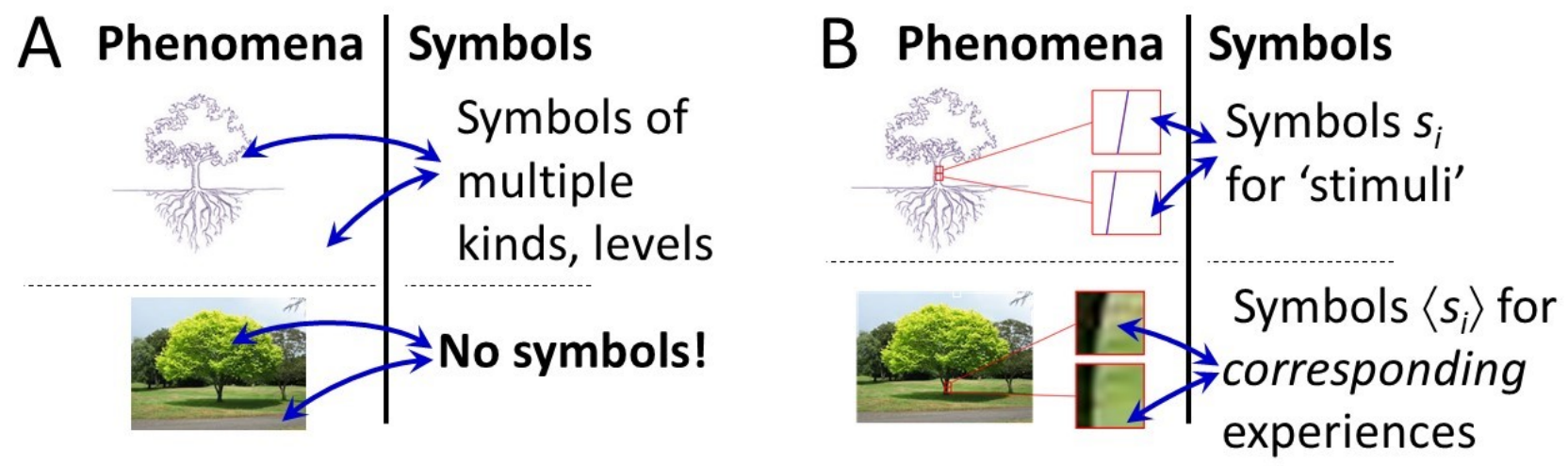
environmental correlate. It can be triggered by a feature of the environment. For example, a cupcake in a shop-window might make me think about dinner. Thinking can be about environmental features. 'I wonder how much that cupcake costs ...'. But thinking about the cupcake in that way is not the same as $\langle$ visual-cupcake $\rangle$. (Note that a full account of angle-brackets symbolism has to account for the different sensory modalities by which we interact with the environment. We might also want to write taste-cupcake, for example.)

So, it takes a little more work to set up angle-brackets symbolism for thinking and feeling. But it's entirely possible. For example, take the thought 'I wonder what that cupcake costs'. If someone says out loud 'I wonder what that cupcake costs', then we have 〈compression-waves-in-the-air-due-tospeaking-'I wonder what that cupcake costs'〉, as an experience of the external environment. The experience 〈thinking-'I wonder what that cupcake costs'〉 can be defined as a neighbour of that experience-of-the-externally-spoken. (If you're thinking, 'why don't we just explain thinking in terms of brain activity', the whole point is to develop a symbolism for experience first ${ }^{8}$. Obviously both external and internal experience have a contribution from brain dynamics. But consciousness science is supposed to explore the relationship between the two! It doesn't do to define part of what we are studying, in terms of another part.)

I mentioned in Chapter 1 that there seems to be a strong human tendency to define consciousness in terms of cognition and sentience. That is, invoking the word 'consciousness' typically leads people to 'I'. And 'I am conscious' means 'I have thoughts and feelings'. This splits the experiential realm into 'I' and 'not I' (e.g. thoughts and feelings, versus environment-originated colours and sounds - see Figure 1.5B). Splitting of this kind has a number of subtle and disastrous consequences. We'll keep returning to these throughout the book, but here are a few headlines. For one, by saying that the experienced environment isn't experience, but 'the world', we lose touch with reality. Physics goes awry. The foundations of science itself become shaky. In addition, we get the phenomenon of conscious experience wrong, throwing away half of it, and making consciousness science impossible. Finally, the split-reality conception doesn't sit well with angle-bracket symbols (which unify all components of experience). And simply, not using symbols makes consciousness science unnecessarily difficult. The simplest way to get at the points we'll need - to get to the One Experiment - is to compare and contrast red and 〈red). When we define consciousness in terms of thinking and feeling alone, we neglect that simple and critical distinction.

\subsection{The Other Side of Ineffability: Mainstream 'Report' and 'Inferred Reconstruction'}

Now, at the same time as the qualitative-ineffability objection (and before the introduction of anglebracket symbols), something I call 'mainstream consciousness science' has been going on, since the early 1990s. (I'll say more about the history and current state of mainstream consciousness science in Chapter 3.) The basic approach of mainstream consciousness science is to measure people's brainactivity while they report on conscious experience ${ }^{9}$. Investigators then use brain-and-report data to try to infer which kinds of brain-activity play a role in which kinds of conscious experience. Obviously, scientists performing these kinds of experiment don't believe the ineffability objection is fatal. And, they see a way around it, without using angle-brackets! What's going on?!

A basic tool in mainstream consciousness science is 'inferred reconstruction'. This simply means, when you report 'I experience red', I reconstruct the meaning of that report based on what it means for me. That is, I know what redness is for me. Therefore, I can understand what experience-of-red is for you, even if I can't explain the difference between experience-of-red and experience-of-green (for me, or for you!). Thus, inferred reconstruction provides a way around the ineffability objection. (Of course, 
what you call redness may be what I call greenness, and vice versa ${ }^{10}$. There are ways round this. For example, we can make an assumption about similar-enough natural systems having similar-enough properties. Or we can construct a science 'up to' the potential swapping of experiential qualities.)

Consciousness science using inferred reconstruction says that qualitative ineffability is not an obstacle, because I can use my ineffables to assign meaning to yours. Essentially, an experimenter is using the reports of participants in experiments to imagine what she herself would experience. If her overall approach is successful, she also discovers brain activity associated with components of her experience. To communicate results publicly, she also constructs some sort of framework in which other people can usefully interpret her work. (E.g. she assumes their experience-of-red is the same as hers. Both are ineffable, but they are the same! It's like saying $l k j f=l k j f$, even if we have no idea what $l k j f$ means, or stands for!)

\subsection{The Role of Angle-Bracket Symbols in Mediating the Ineffability/Report Divide}

Perhaps you already see that angle-bracket statements such as $\langle$ red $\rangle$ depend on some sort of inferredreconstruction context. That is, red is an objective symbol - it's the same for all people, as it labels some wavelengths of light, in the unseen-realm. Implicitly, $\langle r e d\rangle$ is then meant to stand for a common experiential quality that occurs when red light hits the eyes of a typical human being. One context in which to state or create $\langle r e d\rangle$ symbols is via a inferred reconstruction that assumes all people's experience of redness is the same as mine. (Other more complicated constructions are also possible.)

Thus, angle-bracket symbols are related to inferred reconstruction. Both approaches reject qualitativeineffability. But - the inferred reconstructionists may say - why do we need angle-bracket symbols? We've already got a method that gets around ineffability. Clearly, however, inferred reconstruction alone hasn't convinced the ineffabilists (to coin a term!). They still believe in the problem of ineffability.

Now of course, we don't need everyone to believe the same thing. But we do need enough people to believe in the same thing to generate actions that serve the wellbeing of humankind. And that's not what we have in the current study of consciousness. This brings us back to the idea of 'condensing divisions'. (Or, said more conventionally, 'creating more consensus than there was before'.) It's not just about creating something that works for me, or for my faction. It's about bringing other people along. (This is where it can get tricky. Perhaps you'll agree that it sometimes seems like certain others just don't want to be brought along, no matter what the evidence ... We'll have a lot to say about this, as we progress.)

In this case, angle-brackets symbols can play a role in 'bringing along' the qualitative-ineffabilists. If their objection is framed in terms of allegedly-irreconcilable difference between quantifiable, measurable wavelengths and non-quantifiable, ineffable redness, the symmetry and relationship between red symbols and $\langle$ red $\rangle$ symbols can help reconcile them to inferred reconstruction.

\subsection{The Other, Other, Side: 'Too Soft for Physics' - Getting Qualia into Hard Science}

Unfortunately, divisions in the study of consciousness are rarely as simple as camp-1 versus camp-2, as the ineffabilists 'versus' reconstructionists example might suggest. Another significant view on the study of conscious experience comes from those physicists who think that inferred reconstruction is 'too soft' for physics ${ }^{11}$ (Figure 2.3A). More generally, there are those who think that introducing 'subjective' things like 'consciousness' into hard science is the end of science itself! (Perhaps some of this reaction is due to poorly-argued popular appeals for an active role of 'consciousness' in quantum

(C) Nicholas Rosseinsky, 2019 
Figure 2.3: Three Major Views and a Role for Angle-Bracket Symbols. Three different views about conscious-experiential science are depicted as three black squares. A. Those who believe that conscious experience is 'ineffable' also consequently believe that consciousness science is impossible. Within the academic study of consciousness, ineffabilists are in an argument with those who believe in 'inferred reconstruction': that I can reconstruct the allegedly-ineffable quality of 'redness' in your report for myself, by referring to my own unspeakable, inexplicable, but nevertheless directly-presentto-me quality of ... redness! Some unquantified (but perhaps large) subset of academic physicists (and other scientists) disagree with the view that inferred reconstruction could be the basis of a so-called 'hard' science of subjective experience - for example, a science whose inferences could in principle have an effect on the foundations of physical theory. B. Angle-bracket symbols can in principle play a condense-and-distill role (Figure 2.1) in the middle of this three-way debate. They can explain to ineffabilists how it is possible to integrate quantitative mainstream science with qualitative features of subjective experience. They can give physicists comfort about the possibility of creating rigorous foundations for conscious-experiential science. And they can offer inferred-reconstructionists a way to explore and establish a valid experimental methodology for their approach (Chapter 4). (Interestingly, it is the inferred reconstructionists who seem most resistant to angle-bracket symbolism, even though at first glance it is a way of affirming and concretizing their approach. Perhaps they see it as an unnecessary complication, when they think a purely-verbal approach is entirely functional. Alternatively, perhaps they intuit that a precise analysis will reveal extreme problems with their current methodological foundations, which will take the One Experiment to fix!)

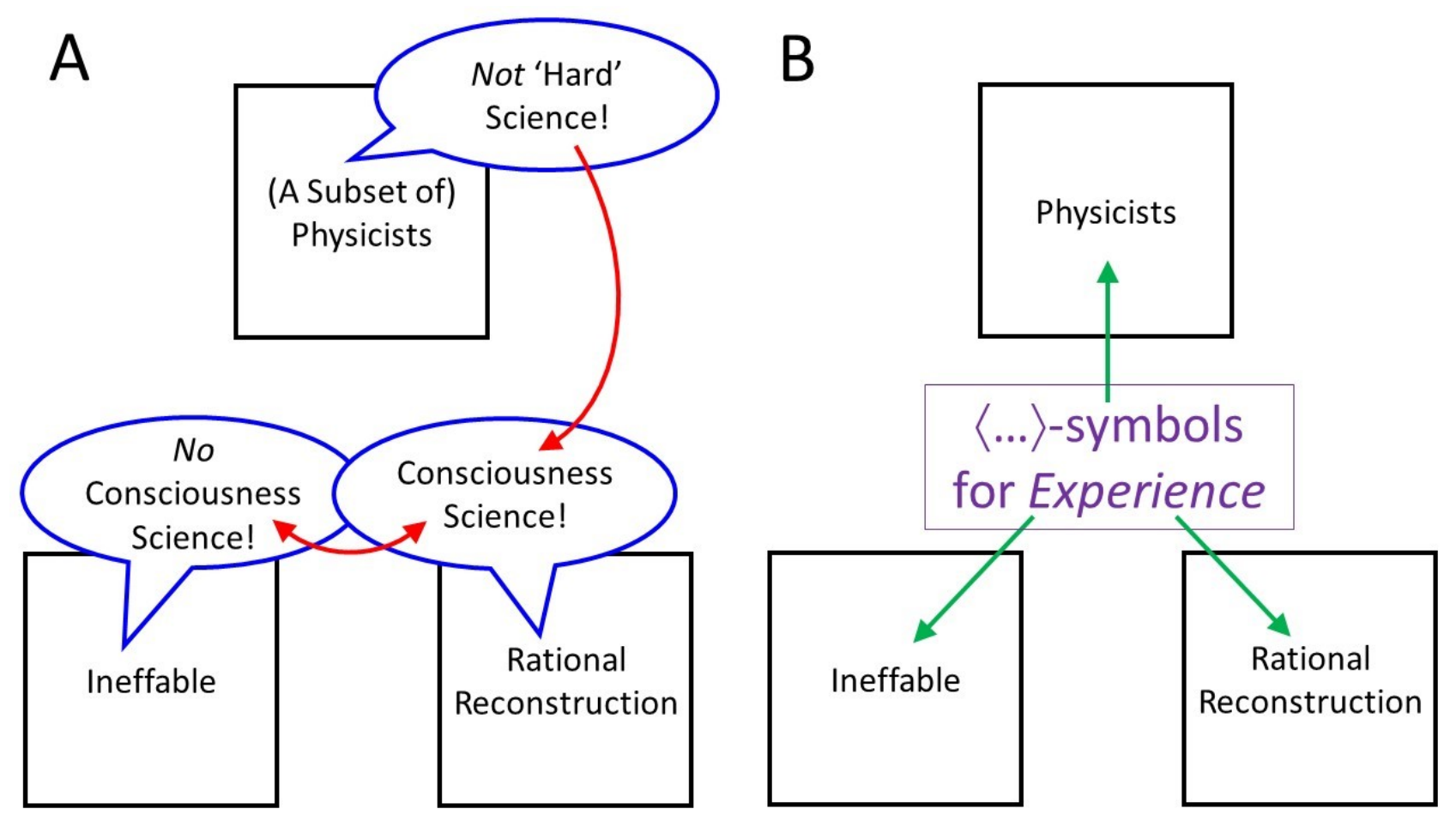


measurement ${ }^{12}$. But it's equally poor logic to say that an appeal to 'consciousness' was misapplied in one area, so we shouldn't study it ever!)

Perhaps the most powerful first-level application of angle-bracket symbols is not actually in the ineffable/reconstruction debate. It might instead be as an antidote to 'consciousness reluctance' in the physics community (Figure 2.3B). The purely-verbal inferred-reconstruction approach can seem wishy-washy to numerically-precise physics. It looks like psychology, rather than physical or biophysical science. And many physicists are frankly skeptical that psychology is a real science. (More politely, they make a distinction between 'hard sciences' such as physics, chemistry and biology, and things like psychology and economics that are at best 'special' sciences!) But the exclusion of physicists from the study of conscious experience is literally a disaster-in-waiting ${ }^{13}$.

The angle-bracket symbolism can be used to state hypotheses about conscious experience in the purelysymbolic, mathematical, format that physics is built around. In a physics-theory context, it's vital to overcome the ineffability argument in a symbol-based rather than verbal form: symbols enable the connection of consciousness science with physical theory. Symbols provide a way to get qualia into hard science. (Loosely speaking, 'qualia' is a philosophical term for the collection of experiential components such as redness, blueness, hotness, middle-C-ness, and so on.) This connection is critical for a whole host of reasons, but primarily because the benefits of consciousness science to humanity depend on it taking us to a to a revisit of our current scientific conceptions of reality and identity. These conceptions have their foundations in physics, so we can only hope to co-explore consciousness, reality and identity in a setting that links the study of consciousness with physical theory. (Another crucial reason for a link-up is to bring the precision and rigour of physics into the study of consciousness. Without the discipline that physics provides, current basic mistakes can keep running, risking all the benefits that a properly-scientific treatment of consciousness would bring.)

Now, angle-brackets symbolism can state theories-of-experience in a way that's familiar to physicists. But physicists can still object to the contextual assumptions that are necessary in setting up those symbols. Recall that I could object to the use of symbols like $\langle r e d\rangle$ by saying 'what if my $\langle r e d\rangle$ is your $\langle$ green $\rangle$ ?'. Even though it is possible to create assumption-free settings that get round these kinds of objections, it can still seem like there's still unscientific subjectivity involved in using these symbols. And again, we hit the point of bringing people along, as well as being right. At some point, it is crucial to bring physics along for the consciousness science ride we're going to preview in this book. If people

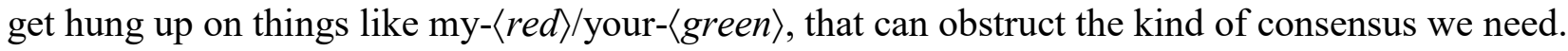

To build enough physics buy-in to the angle-brackets approach, we may need another level of argument, to motivate engagement. This other level has to do with pointing out science's own needs for a scientific analysis of subjectivity. Currently, the playing-field is inappropriately tilted towards the exclusion of the subjective (for reasons we'll discuss later). Recognizing how the inclusion of a precise and rigorous treatment of subjective experience can help science stabilize itself in the face of today's post-Truth attacks will rebalance things.

\subsection{Science-as-a-Whole Needs Qualia and Subjective Experience!}

Let's recap, briefly.

Science-as-a-whole is currently confused and ambiguous about the unseen/experiential distinction. This is reflected in Chapter 1's everyday usage of 'consciousness' to label some combination of the reflexive-self ('I'), and thoughts ('my intelligence') and feelings ('my sentience'). This usage divides up experience into inner-related (thoughts and feelings) and environment - and discards the 
environmental component from experience! (Scientists are everyday people too. They can also inadvertently slip into using the definition in Figure 1.5B! Or maybe they were never taught that Figure $1.5 \mathrm{~B}$ is problematic ...) When we do this, we make self, thoughts and feelings 'subjective', and we can believe that environment is not experience - it's 'objective'! That is, we can believe that 'experience of table' is actually the table, not an experience.

All this we've seen before. Let's now make the specific 'experience' not a tree, or a table, but a science lab, with apparatus and measurement-instruments (Figure 2.4A).

Now, when we go into a science lab (as scientists), we may believe that our interactions with apparatus and measurement devices are all purely objective. But this is clearly wrong. Just we never see a table directly, so we never see a particle-collider or gravimeter! When we do experimental science, we need to make explicit assumptions about the relationships between experience-of-apparatus and actual, objective, apparatus (Figure 2.4B). Moreover, we need to make explicit assumptions about whether it's the experiential or objective apparatus that we actually think scientists are basing science on! (This may be a bit obscure at this point. All will be made clear!)

Next, setting aside confusions about the science-lab external-world, it's obvious that scientists use subjective capacities when creating theories, looking at data, and reviewing other people's papers. How can we attribute reliability to that use of subjectivity, without a scientific theory of it?! You may say 'It's obvious when something is logical or not logical' - but logical/not-logical assessments take place in a mind that is definitively subjective! It seems contradictory to say 'Science can't study subjectivity, because that's too soft. But it can use subjectivity in each and every assessment that's made in allegedly-"hard" sciences!'

Finally, science needs to define its own non-angle-bracket symbols, for example symbols like ' $e^{-}$' for an electron. (If you are familiar with physics, try doing that right now, without making any reference to aspects of your experience - which of course includes experience of objects like tables, trees, and computers!) To make those symbols relevant to the Universe we inhabit, science has to say something like ' $\ldots$ and those electrons are constituents of things such as tables that then appear in experience'. If physics doesn't refer to experience when it defines its own basic symbols, it creates a theory of a purely-imaginary Universe, which may or may not have anything to do with our Universe! (You may say, 'But experimental predictions show that this imaginary Universe is exactly the same as ours!'. However, if physics is forbidden from speaking about experience, because of its own 'standards', it can't compare theory to experiment - because experiment involves experience! ${ }^{14}$ )

These three arguments point out the role of the subjective/objective relationship in carrying out scientific experiments, in scientists' mental activities, and in the definition of symbols and constructs that are at the foundation of science itself ${ }^{15}$. Of course, it's possible to make vague and generic assumptions that ensure the integrity of science despite the universal use of subjective aspects. (For example, 'we assume scientists' subjective cognitions are collectively reliable'.) But we can't assume away the relevance-problems for physics (the third and final observation, above), if physics actively excludes conscious experience. And it's precisely the claim of assumption-free precision and rigour on the part of science that is supposed to be the basis of its reliability. Conversely, the need to make assumptions - or a persistent confusion that obscures this real need - weakens science. It's precisely these sorts of weaknesses that are the loopholes through which post-Truth attacks gain their intellectual vigor. 
Figure 2.4: Is Data from Scientific Experiments Collected in Experiential or Unseen Realms?! This Figure depicts particle-track data of the kind used in establishing the existence of the Higgs boson in 2012 (red-orange-blue lines and circles on a black background, upper-left) and the tunnel containing the Large Hadron Collider (blue tube in a white-grey tunnel lower middle) that was the central experimental apparatus in Higgs-detection. Science never really examines or answers the question: is science being done in the experiential-realm (left-hand side, coloured images) or in the unseen, 'objective' realm (right-hand side)? Either choice - without a precise, rigorous of the relationship between the two realms i.e. a proper conscious-experiential science - seems to lead to profound, perhaps even fatal, problems for scientific claims to rigor and relevance.

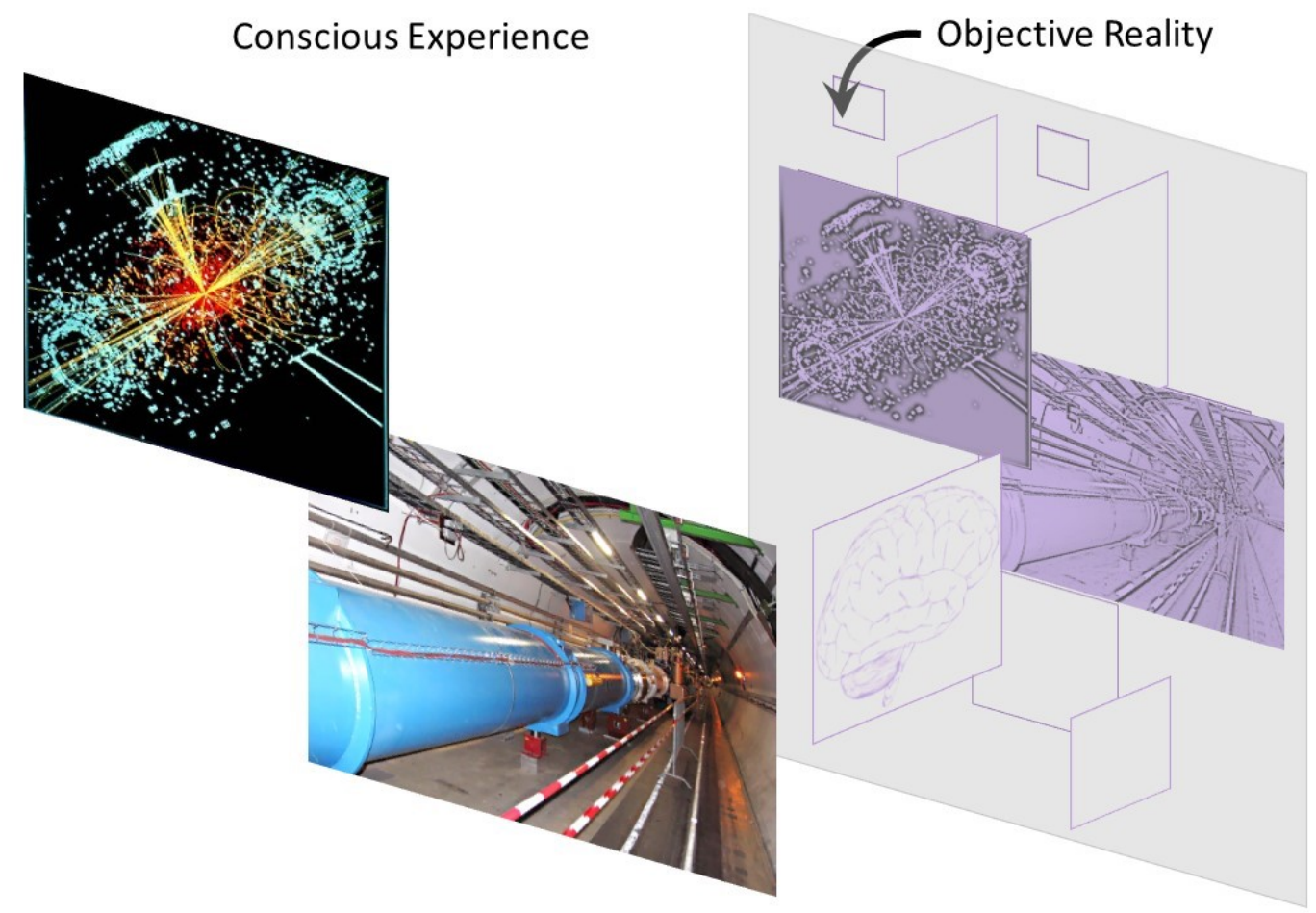


How does all this relate to our main thread, about 'effing' consciousness and getting qualia into hard science? I said physics may resist angle-brackets symbols, really on no very good grounds. We then point out 'Angle-brackets symbols can help science treat subjectivity rigorously, and thus establish rigorous responses to the three subjective/objective problems for science-as-a-whole'. A few years ago, science's inattention to subjective/objective issues in its own foundations and conduct might have been OK. But if science is to regain a solid footing in a post-Truth world, it needs to fix these issues. Anglebracket symbolism - as part of a true science of consciousness - are crucial to that fix. This observation must far outweigh any subjective (!) reluctance in physics concerning things like my- $\langle$ red $\rangle /$ your$\langle\text { green }\rangle^{16}$.

\subsection{Interlude: Developments So Far as a Micro-Example of Parts 2 to 4}

We've seen three different views related to qualitative-ineffability: ineffability prevents consciousness science; inferred reconstruction gets around ineffability; and, inferred reconstruction is too soft to be valid physics. I explained how angle-bracket symbolism may help to bring all three factions together. Additionally, I pointed out that a supplementary argument about science-as-a-whole's broader needs for good consciousness science may be necessary, to get physicists on board with some of the necessary assumptions or complications.

The style of approach exemplified here identifies a number of viewpoints related to a single issue, and tries to create a consensus, by theoretical analysis. The specific 'single issue' in focus in this Chapter has been whether qualitative aspects of conscious experience can be integrated with the quantified nature of hard science. Two different types of theoretical analysis were applied: a local, specific, reasoning about consciousness science ('we can use angle-bracket symbols') and a global, science-asa-whole argument ('science-as-a-whole needs a science of consciousness, if that's possible to construct rigorously'). This application of multiple lines and styles of reasoning to bring together a number of contradictory viewpoints is a basic motif in the strategic approach that I'll lay out in the next Chapter.

However, if we could get to where we need to go just by building consensus through theory and reason, there'd be no need for the One Experiment! The role of experiment is precisely to resolve those issues that can't be tackled through theory alone. So, to finish up this Chapter as a micro-example of the book's whole strategic approach, I'm going to sketch how the angle-bracket response to ineffability takes us to a frontier that can't be resolved theoretically. In fact, it takes us right to the One Experiment. (Although, we'll have to approach the One later via a number of routes, and considerably more slowly, to fully appreciate it!).

\subsection{Consciousness Science and Computational Neuroscience: Related, But Not Identical!}

In addition to the mis-definition ${ }^{17}$ of consciousness solely in terms of thinking-and-feeling, there's another common tendency that can obstruct advances towards a true science of subjectivity. It can be easy to confuse advances in computational neuroscience with advances in consciousness science. (This can particularly be the case if we define consciousness in terms of 'access' - more or less, the ability to report on something.) Computational neuroscience explains how the brain encodes information like 'there's a red patch at such-and-such a location'. It explains how the brain uses encoded information to make decisions. And it explains how decisions - seen in terms of neural activity - are then transformed into behaviours, like coordinated muscle movements. It doesn't explain anything about the subjective experience associated with any of the encoding, decision, or behaviour phases!

To keep that distinction clearly in front of us, I sometimes use a notation that explicitly differentiates between experience-of-red and encoding-of-red. Of course, experience-of-red is written as $\langle r e d\rangle$. In 
this book, I'll write 'encoding of red' as Enc[red]. ('Encoding' of red means certain neurons get active in certain ways, when red is presented at a certain location in an experiencer's surroundings. When blue is presented at the same place, there's a characteristically-different pattern of neural activity. In principle, with complete knowledge of the "neural code' ${ }^{18}$, we could use full knowledge of brain-state to deduce the complete sensory environment.)

Now, it may be completely unclear to non-specialists that understanding of how brains encode red is not the same as understanding how experience-of-red occurs! (To be fair, it also seems that this point is sometimes unclear to specialists!) After all, we could reason like this. 'Brains are involved in experience. Experience-of-red depends on encoding-of-red. The simplest theory-of-consciousness is then that brain-encoding-of-red is the same as first-person-experience-of-red.' That is, if I find out what neural activity carries out Enc [red], then I've also discovered how $\langle$ red $\rangle$ comes into existence. The neural activity that implements $E n c[$ red $]$ also 'is' (or 'creates', or 'causes' ${ }^{19}$ ) $\langle r e d\rangle$.

If it's hard to see what's wrong with that - don't worry! The 'identity theory' of consciousness is essentially the idea that encoding-activity in the brain is conscious experience. And this was a major theory for a good part of the twentieth century. It still survives in a healthy way today. In fact, we'll have to spend a good deal of time deconstructing the identity-theoretic viewpoint, to get through some of the obstacles to the One Experiment!

So, what is wrong with 'theory-of-encoding equals theory-of-conscious-experience'? Well, there are all the sorts of things that philosophers like to get excited about, such as correlation doesn't mean causation, and correlation doesn't mean identity. As we'll see, in the current mainstream, people tend to sidestep these kinds of problems. These sidesteps are usually equivalent to additional assumptions (although they're not usually documented in this way). And many of the additional assumptions are more or less OK. (That is, they don't introduce later contradictions or inconsistencies. They are, of course, still extra theoretical baggage!)

But, beyond philosophical critiques, there are two far more critical and inter-related points. The first is that there are many different places in the brain that encode a simple thing like red at such-and-such a location. Presenting red in someone's visual field leads to a successive series of neural activities in a hierarchy of brain areas. Which of these many encoding activities are involved in or associated with (or 'identical to') $\langle r e d\rangle$ ? Relatedly, say there was just one location and one local activity pattern that encoded $\langle\mathrm{red}\rangle$. (This is now a sort of imaginative 'thought experiment'.) Does localized activity at that one location necessarily cause or instantiate or associate with $\langle$ red $\rangle$, under all conditions? (For example, say that one location was artificially stimulated, when I'm in deep, dreamless, sleep.) Or does there need to be some sort of general background brain-activity, without which localized activity doesn't lead to experience? These sorts of issues and arguments establish a first point: there's more to a theoryof-experience than simply establishing a complete theory-of-local-encoding. Consciousness science isn't just computational neuroscience.

The second point is extraordinarily obvious, and extraordinarily overlooked. You and I are having a conversation about conscious experience which presumes that I can meaningfully write about it. Or speak about it. That is, we are presuming that my writing something like 'experience-of-red' is scientifically meaningful. If it isn't, then $\langle$ red $\rangle$-symbols are scientifically meaningless. Then, a theory of encoding can't be a theory of experience, because we can't have a scientific theory of experience of any kind. 
At this point, you may be very confused! After all, didn't I introduce angle-bracket symbols precisely to make experience 'scientific'? How could they be 'scientifically meaningless'? Is Nicholas now taking an ineffabilist position?! The issue is that we need to take great scientific care with what we are doing - if we want to do science!

I can write $\langle r e d\rangle$. Or I can say 'I experience redness'. But what causes or leads to that act? Does experience-of-redness itself actually directly participate? Or, is the brain generating the experience, and, more-or-less independently, generating the speech act (or the symbol writing)? If it's the latter, isn't it then that experience and report have a common cause, but aren't themselves causally-linked? (See Figure 2.5.) And doesn't that then mean that report isn't really about experience, directly?

Frankly, Figure 2.5 is philosophically and scientifically naïve. Experts laugh at it. 'It's not like that, at all'. But as we'll see, what we really have in the mainstream right now is a sort of Emperor's New Clothes. Experts are right. It's not like Figure 2.5 (probably!). However, the naïve questions posed by this picture haven't been properly answered by the sophisticated explanations of those same experts. If experience doesn't play a causal role in report-of-experience, can we use report-of-experience in a scientific way? (For example, how can I write $\langle$ red $\rangle$ to scientifically report my experience-of-redness?) But if experience does play a scientifically-sufficient causal role in report, shouldn't we be able to see its causal activity in brain-dynamics?! And wouldn't that causal coupling between experience and brain-activity be a fundamentally-new kind of observation?

These questions characterize a second way that consciousness science is not computational neuroscience. Just now, we saw that local encoding isn't necessarily identical with experience, so a theory-of-encoding isn't a theory-of-experience. In these two questions, we've come across a completely independent and far deeper problem. Consciousness science has to construct a methodology that explains how we can report experience. You may think this is straightforward. 'I experience redness, and that experience of redness causally participates in my report "I experience redness".' But a tension comes from the fact that consciousness science is built on top of physics. And physics already has a complete explanation for your report: brain-dynamics did it! Experience didn't play any role, according to standard physics - because standard physics doesn't even recognize experience, as a thing ${ }^{20}$ ! Looked at this way, brains cause experience, and brains cause report. Experience itself plays no direct role in report-of-experience! This turns out to be an insurmountable problem for consciousness science under orthodox physics, as I'll explain in later Chapters.

Now, we will spend a lot of time in the following Chapters looking at how mainstream consciousness science tries to get around this problem. It tries to do something that's actually impossible - keep to a standard-physics foundation, and have report-of-experience be caused by experience. It's a delusional insistence that these two things are simultaneously possible that's the primary obstacle to even discussing the One Experiment, within the field of consciousness science ${ }^{21}$. In this sense, mainstream conscious-experiential science in its current form is an active obstruction to science-3.0.

\subsection{Introducing a New Division - And Testing It Experimentally}

We saw that - at least, in principle - angle-brackets symbols could create consensus between the ineffabilists, reconstructionists, and the too-soft-for-physics camp. But now I've hinted at another division. If we keep standard physics fixed, we can't write angle-bracket symbols in a scientificallymeaningful way. (I haven't 'proved' this point, although perhaps you can see that the reasons for inability to do good report-based experiments leads to problems for symbol-definition. I'll 
Figure 2.5: What is the 'Causal Source' of Reports about Experience? The causal origin of reports about experience is a vital issue for the foundations of consciousness science (and so for science 3.0, and the future of humanity!). A 'report about experience' is something like 'I experience brownness of that table-top'. Of course, we can program a computer to speak in the same way, when it detects brown (in the unseen realm!). (Recall that italicized brown refers to brown-wavelength light, an unseen, objective-realm thing.) A: Brain activity causes report-of-experience. One possibility is that our reports are like a computer's! Our brain more-or-less mechanically says 'brownness' - but that report is really driven by brown light and its neural consequences. At the same time, we have an experience $\langle$ brown $\rangle$, and we tell ourselves that report was directly driven by, sourced in or caused by experience. If the causal chain is light-to-eye-to-neural-activity-to-speech-muscles - without experience-itself playing any part - we're deluding ourselves. One curious consequence of this picture is that it seems impossible to ever talk meaningfully about experience - but we do it all the time. (I just did!) But there may be some even deeper delusion at play. B: Experience causes report-of-experience. This actually requires experience to couple with, or transfer information into, brain-dynamics governing report. (This step is not shown.) Experience-causing-report accords with intuition, but turns out to conflict with standard physics (Chapter 4).

Philosophers and others will criticize various aspects of this simple, introductory picture ${ }^{22}$ (and associated language). Notably, one school ('identity theory') tries to claim that the distinction between $\mathrm{A}$ and $\mathrm{B}$ is meaningless, because conscious experience is nothing but, and identical to, brain-activity that 'causes' it (by virtue of identity!). But this view is at least puzzling. Even if experience were folded into brain-activity, with no intervening machinery, such brain-activity would seem to have two different kinds of properties, at least: its conventional, unseen-realm aspect (molecules, electromagnetic field states etc.,), and its experiential, qualitative aspect (e.g. $\langle$ brown $\rangle$ ).
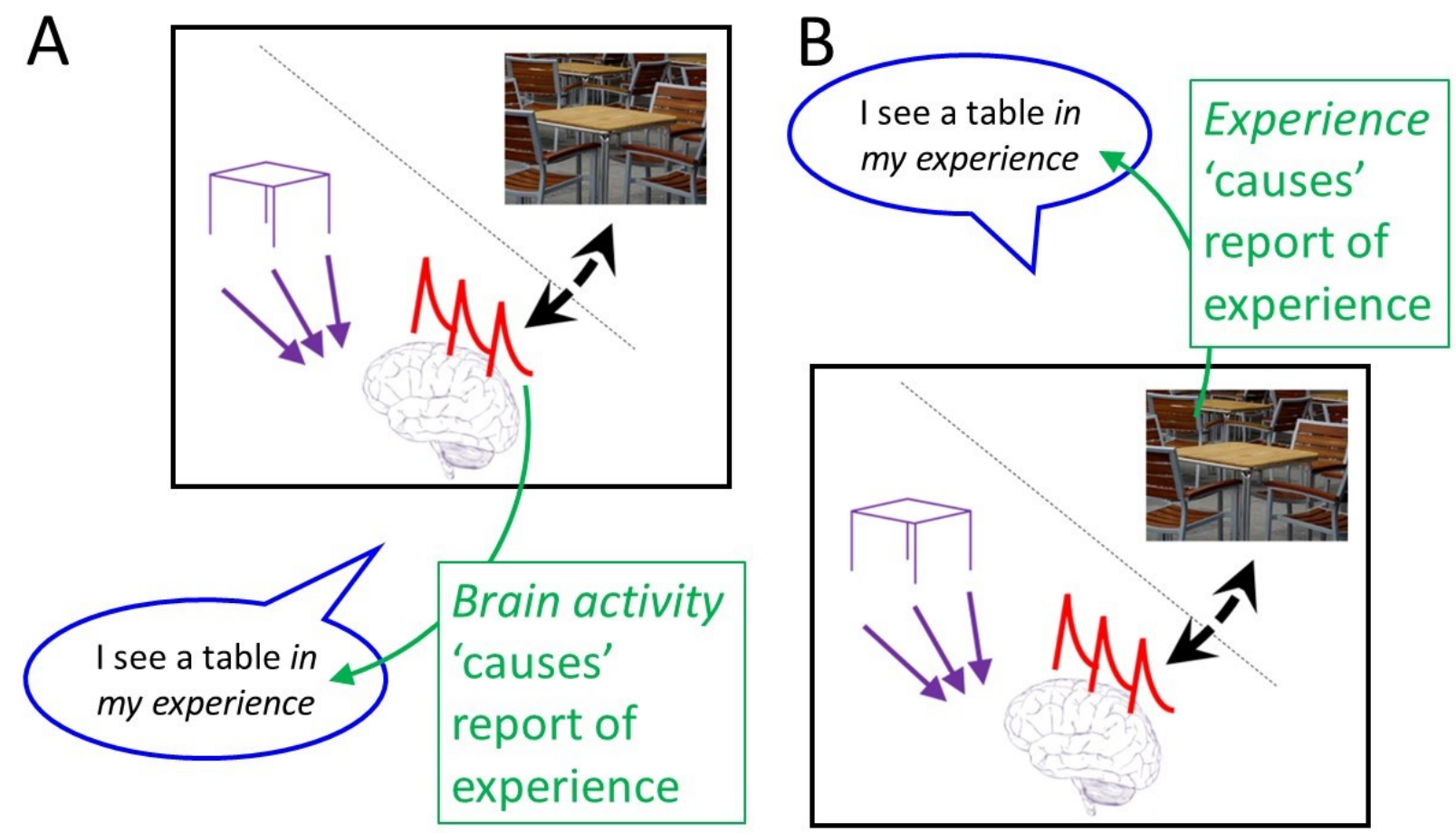

(C) Nicholas Rosseinsky, 2019 
get to all this in more detail, in Chapter 4. I'm really just previewing forthcoming attractions, right now!)

Now perhaps you expect me to argue either that we must keep standard physics fixed - because, you know, physics is the foundation of science. (Which would mean that our carefully constructed threecamp proto-consensus falls apart. Standard physics means 'no symbols' means no peace-making!) Or, maybe, that 'we have to change standard physics, so we can do consciousness science, and get all the benefits we've touched on in this Chapter and the last'.

Actually, neither! What we need to do is an experiment, to test the physics involved in the report of experience (Figure 2.6). If standard physics holds, experience doesn't play a causal role, and we can't have consciousness science. (If standard physics holds, inferred reconstruction is an illusion as a scientific method.) If a specific kind of non-standard physics occurs, in which certain information transfers from experience to brain-activity, then it's possible to construct a rigorous consciousness science. (Further experiments and theorizing will be necessary. But without that non-standard activity, no consistent, rational approach to consciousness science is possible.)

Where should we look for this 'non-standard physics'? In the brain, of course! The question will be whether brain-activity follows standard physics all the time. Or whether information-transfer from conscious experience violates (or better, extends and expands!) standard physics. Excitingly, we may already have collected experimental data that demonstrates non-standard brain-physics. It's just that no-one has done the required analyses yet ${ }^{23}$.

Why not? Because of the troubles with consciousness science ...

\subsection{Summary}

Three important factions disagree about whether we can create a science that integrates subjective, qualitative things like redness with objective, quantitative things like red (red-wavelength light):

1. One school of ineffability claims that redness (qualitative) is so fundamentally unlike red (quantitative) that we'll never be able to put the two together.

2. Inferred-reconstructionists use your report of an 'experience of redness' to infer the qualities you're experiencing. They claim to collect data on brains and on (reconstructed) experience, simultaneously.

3. Some physicists think that reconstructing experience from verbal accounts is too 'soft' and nonphysicsy to qualify as hard science. They want to keep physics separate from consciousness 'science'.

Angle-bracket symbols (D2) are a way to bring subjective components of experience into hard science (to 'eff' the ineffable!), and create some consensus amongst (some members of!) these three camps.

Remaining (e.g. 'my- $\langle$ red $\rangle /$ your- $\langle$ green $\rangle$ ') resistance from physicists can be met by pointing out the importance of consciousness science to science-as-a-whole, which uses subjectivity in three ways:

I. When apparatus is used and measurements are taken, subjective experience is necessarily involved. Is it experience-of-a-measurement or unseen-realm-measurement that's recorded in a scientist's brain?

II. When assessments of theories and data are made, and new hypotheses created, subjective mind is necessarily at play. Is 'subjective seeing of logical truth' functioning in science, or not? 
Figure 2.6: The Entirety of Conscious-Experiential Science (including New Symbols and 'Condense/Distil') is Contingent on Results from the One Experiment. Note that the 'motif' inside the black-rectangle at the upper-left reproduces Figure 2.3B: the use of angle-bracket symbols to create working-consensus between three views. Also, the central movement of this Figure - the purple arrow leading from the set of black squares representing different views to an experiment comparing redrectangle/green-rectangle stances - reproduces Figure 2.1A. Thus, this Figure begins in the top-left by emphasizing that angle-bracket symbols introduced in this Chapter (Figure 2.2) are a crucial tool in the condense/distil movement. The large black-rectangle in lower-middle offers new clarification of the goal and content of the 'Experimental Test': it is to arbitrate between hypotheses about 'cause' of report-of-experience (Figure 2.5). (Note that framing the alternatives as 'experience causes ...' or 'brain causes ...' is a simplification-of-language. As hinted at by the asterisked text, the contrast is between a non-standard physics that couples experience with brain-activity, or a standard physics that does not. See Chapter 4.) If experimental data indicates experience doesn't couple with brain-activity, reports are not meaningfully about experience. (Even the word 'experience' in the previous sentence is dubious!) We follow the red arrow: conscious-experiential 'science' does not exist, and even the declaration of angle-bracket symbols in the top-left was a mistake, or delusion. Alternatively, if experience does couple in specific ways, speaking about experience is meaningful, defining and using angle-bracket symbols was a valid step, and the experiment itself (large-black rectangle) was also valid.

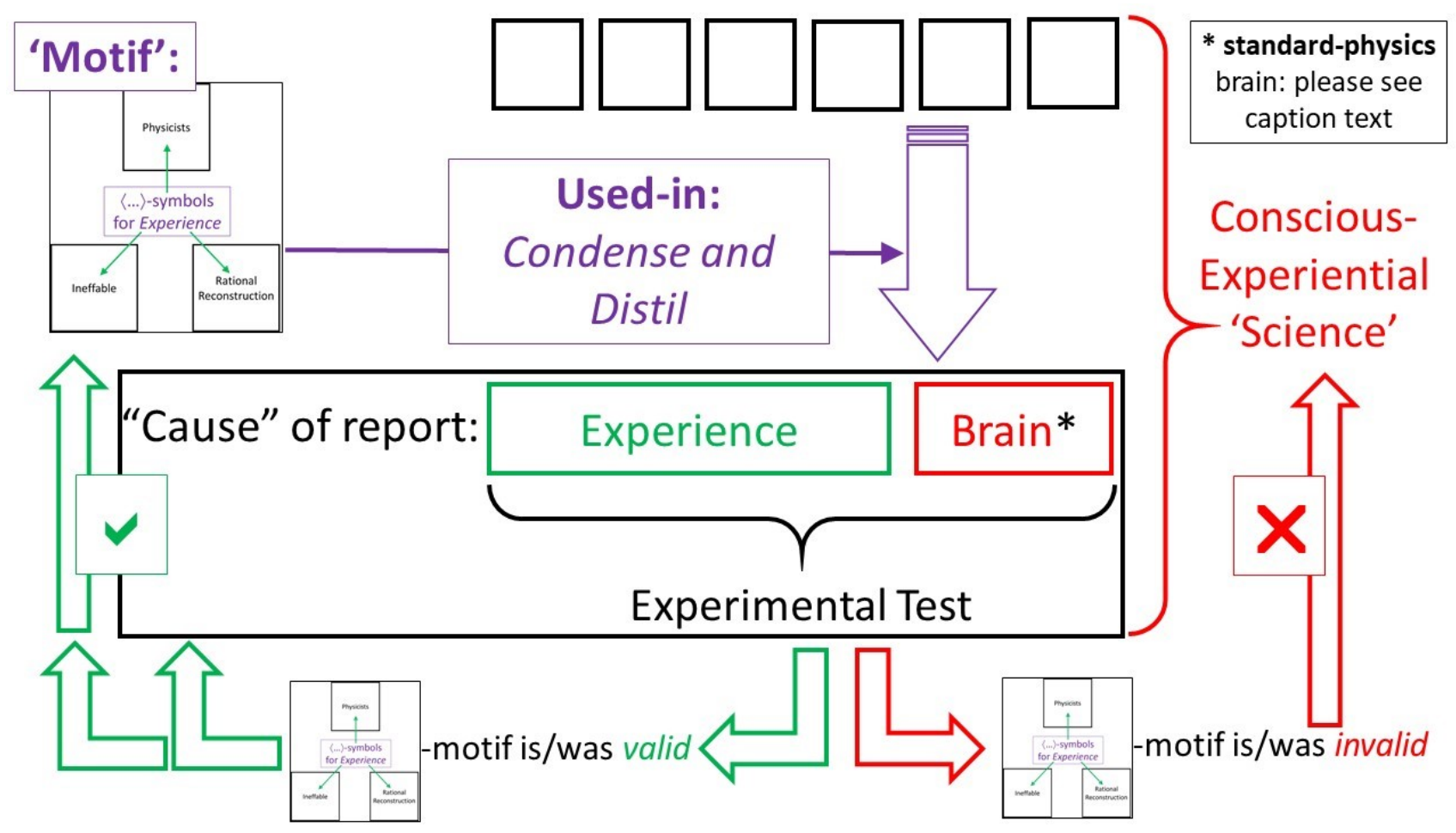


III. When scientific constructs like 'an electron' are defined, they must either be linked to subjective experience (of objects and apparatus), or not. If not, physics seems to be about an imaginary Universe.

Angle-bracket symbols can help with 'condensing' factions, exemplifying the strategic use of theory. But not every difference can be resolved by theoretical argument. Sometimes, we need experiments.

Computational neuroscience is not the same as consciousness science. Obviously, for any experiment to be about conscious experience (and not just encoding), it must collect data about experience!

A central and overlooked issue is whether we can collect data about experience. If experience doesn't causally participate in report, it seems reports aren't scientifically-reliable data about experience.

The One Experiment tests whether experience does causally participate in report. Thus, it tests whether we can have a science of conscious experience. As an experiment that determines the scientific viability of all other conscious-experiential experiments, it is the ultimate end of the proto-scientific phase.

\subsection{Further Reading}

The definition and application of angle-bracket symbolism is discussed at length in Consciousness Science: A New Approach (Rosseinsky, 2019) A technical discussion in a physics-theoretic setting can be found in Installing Physical Theory's Missing Symbolism for Conscious Experience Reveals Ten Puzzles for Science as a Whole (Rosseinsky, 2018a). Problems for mainstream consciousness science arising from the causal role (or not!) of experience in report will be the main theme of Chapter 4. If you want to go to directly to a more technical treatment, please see One Experiment to Start Them All: Consciousness Science's Missing Foundation (Rosseinsky, 2018b). (Consciousness Science: A New Approach also discusses report, causality, and the One Experiment. As will usually be the case, its discussion is at a level between the introductory, non-specialist, treatment in this book, and the more advanced, technical treatment in standalone papers.) 


\section{References}

Goff, P. (2017). Can Science Explain Consciousness? Philosophy Now 121, Aug/Sep 2017.

Penrose, R. (1989). The Emperor's New Mind. Oxford: Oxford University Press.

Penrose, R. (2000). The Large, the Small and the Human Mind. Cambridge: Cambridge University Press.

Rosseinsky, N. M. (2018a). Installing Physical Theory's Missing Symbolism for Conscious Experience Reveals Ten Puzzles for Science as A Whole. doi:10.13140/RG.2.2.36652.31361/1.

Rosseinsky, N. M. (2018b). One Experiment to Start Them All: The Missing Foundation of Consciousness Science. doi:10.31234/osf.io/zgb9w.

Rosseinsky, N. M. (2019). Consciousness Science: A New Approach. www.thescienceweneed.com.

Rosseinsky, N. M. (2020). Reclaiming Reality. www.thescienceweneed.com.

Tegmark, M. (2000). Importance of quantum decoherence in brain processes. Physical Review E 61, 4194.

Tegmark, M. (2015). Consciousness as a state of matter. Chaos, Solitons \& Fractals 76, 238-270. 


\section{Endnotes}

${ }^{1}$ In Chapter 1, I wrote mainly about the relationship between science and its post-Truth/'fake news' critics. In Part 5 (Chapters 10 to 12), I'll expand on the impact of proper consciousness science, and its science-3.0 consequence.

${ }^{2}$ As an advanced point, what I'll end up calling the qualitative-ineffability problem (the subject of this Chapter) can be seen as connected to what might be called the dynamical-ineffability problem. (Chapter 4 - the heart of the One Experiment). Namely, if experience doesn't couple with braindynamics in specific ways (dynamical ineffability), then practically it's also qualitatively-ineffable, because it's just flat-out 'unspeakable'. So the two sorts of ineffability aren't always entirely separate, or separable.

3 (Goff, 2017)

${ }^{4}$ The use of italicized words for unseen-realm quantities does introduce ambiguity, however, because I also italicize other words for emphasis. (As I just did with 'does' and 'other'.) That's one reason why science uses dedicated symbols! In Consciousness Science: A New Approach, I use the more precise, conventional, dedicated-symbol approach. In this book, I hope that context will always make clear that 'red' is italicized because it's an unseen-realm quantification, and 'does' was italicized for communicative emphasis.

${ }^{5}$ For a more detailed and complete treatment of angle-bracket symbols, please see Consciousness Science: A New Approach.

${ }^{6}$ Angle-brackets symbolism has nothing at all to do with bra-ket notation in quantum theory that happens to use the same symbols ' $\langle$ ' and ' $\rangle$ '. In quantum theory, those symbols only occur in the forms $\langle a|| b$,$\rangle , and \langle a \mid b\rangle$. Both formally/notationally and in meaning/usage, $\langle s\rangle$ is completely and totally separate and independent of those quantum-theoretic notations.

${ }^{7}$ I would distinguish bodily feelings from emotional feelings. If my arm feels warm, there's an objective (non-brain) condition that leads reliably to a brain-state associated with experience-of-warmth. So bodily feelings have an objective (non-brain) correlate (unsurprisingly, in the body!). While, of course, many different, objective, bodily and external states can contribute to emotional feelings, there's no inherent, stable map (other than the physical brain itself!) from those objective states to experienced feelings. One day, feeling cold might contribute to my sadness ('I don't have enough money to pay the heating bill!') but another day it might contribute to my happiness ('I love playing in the snow!'). The point is that the map from objective-state to experience for emotions apparently depends solely on the brain, whereas for bodily feelings it is from body-state first (and then to brain, and then to experience).

${ }^{8}$ For those familiar with the term, what we are basically doing with angle-bracket symbols is setting up a primitive phenomenology and using it to give meaning to a new set of scientific symbols.

${ }^{9}$ Even so-called 'no-report' paradigms depend on some sort of report, even if it's just 'the report the experimenter believes would occur'. Otherwise, there's no experience to correlate with brain-data! The main point of 'no-report' paradigms is simply to try to avoid mixing brain-dynamics-due-to-the-actof-report in with brain-dynamics-involved-in-experience.

${ }^{10}$ The problem of 'inverted qualia'. 
11 See, for example, Stephen Hawking's skeptical comments about reasoning from consciousness science to fundamental physics, in (Penrose, 2000).

${ }^{12}$ I'm not arguing for a view either way about a putative role of consciousness in quantum state-vector reduction (the 'collapse of the wavefunction'). I'm just noting that a 'consciousness must ...' argumentstyle has landed poorly with a large number of scientists.

${ }^{13}$ Of course, there are a few physicists who have set foot in the consciousness territory, Roger Penrose [The Emperor's New Mind (Penrose, 1989)] and Max Tegmark (Tegmark, 2000, 2015), to name just a couple. But the vast majority seem to think consciousness is not an appropriate topic for physics. Typically, when 'consciousness' is allowed into physics, its definition is limited to cognition or intelligence (or, at the very limit, forms of sentience). These kinds of definition neglect conscious experience, which is the critical issue both for the post-Truth conflict and for the transition to science3.0. (Sentience - properly interpreted - is inherently experiential. But it neglects experience of surroundings, so it's only a partial admission - and that partial view isn't enough to take us to science3.0.)

${ }^{14}$ It's possible to construct a theory of science in which all scientific processes are taking place in the unseen realm, and they all happen to work out perfectly, and experience is irrelevant to the conduct of science. But this is not consistent with everyday practice in which we believe things are true by virtue of experientially-seeing them! In a more nuanced point, scientists depend on 'subjectively seeing the coherence of theories and data' in science. Thus, there's seemingly an explanatory gap, between that observation and a theory-of-science proceeding totally in the unseen-realm, which denies subjectivity any role.

15 These three problems occur immediately and necessarily after we make a clear unseenrealm/experiential-distinction. Plausibly, this is one reason why science as a whole seems to treat this area in a vague and disinterested way: as soon as it's done well, it becomes problematic. But it's wrong to shy away from problems, in part because - as in this case - they often become opportunities. Treating the unseen/experiential distinction properly leads to the One Experiment, and to science-3.0. (And even when problems don't become opportunities, simply burying them because they are problems compromises integrity.)

${ }^{16}$ This comment is not an appeal to relax rigor, in order to patch over foundational problems. It's an appeal simply to examine empty objections to angle-bracket symbols. Once empty objections are seen to be empty, they will be relinquished.

17 'Mis-definition': in a scientific context, if we want to connect coherently to modern physics. There's nothing wrong with everyday usages and definitions, applied coherently in their own contexts.

18 There's some debate about whether a 'neural code' actually exists, in a rigid and stereotypical way. Nevertheless, it seems clear that each human must have an organized and patterned way of converting incoming information into decisions and behaviours. I use the word 'code' to refer to this organized information processing. Notably, for there to be a stable, reliable subjective experience of environment, there first needs to be a stable, reliable brain-representation of environment!

${ }^{19}$ In this specific context - the examination of 'identity theory' - by 'create' or 'cause', I mean, create or cause by virtue of identity-with.

${ }^{20}$ In the next Chapter, we'll examine the 'emergent property' explanation of conscious experience. Adherents of that explanation may say that my claim 'fundamental physics doesn't recognize experience' (so that it can't participate in physics-theoretic causality) is a bit like making an analogous 
(and false!) claim about temperature, say. (Temperature is an emergent property, uncontroversially.) Certainly, fundamental physics doesn't recognize temperature. But that doesn't mean temperature can't have physics-theoretic causality! (Obviously!) So what's the difference between conscious experience and temperature? Essentially, temperature is nothing but a collection of specific fundamental states, and can be said to have the same causal powers as that collection. ('Nothing but' is the same as 'another name for'.) But it's entirely obvious that experience isn't nothing but physical states. Not in the sense that there needs to be some additional substance involved, or some non-physical or supra-physical mechanism at play. Just in the sense that e.g. there's the brain-activity that gives to experience-ofredness (characterized as electromagnetic-field states, say), and there's the experience itself. The electromagnetic field-state is in the unseen-realm, and experience-of-redness is in the experiential realm. Experience may be contingent_on brain-activity, but it's not 'another name for it' ('nothing but' brain-activity). So, experience isn't emergent in the same way that temperature is. And causal reasoning about experience diverges from causal reasoning about temperature. We'll go over this more, in the next Chapter.

${ }^{21}$ As we've seen there are other obstacles outside the field. For example, the skepticism of physicists that consciousness can be studied scientifically. Resolving the causal role of experience in report is part of deconstructing physicists' objections. Other lines of strategic intervention are necessary too, though, as discussed earlier in this Chapter, and developed further in the next.

${ }^{22}$ Contrary to some claims, this Figure does not assume or imply dualism of any kind. For example, I could interpret panel B in an identity-theoretic manner, noting in particular that the dashed-black doubled-headed arrow denotes association between brain-activity and experience.

${ }^{23}$ We'll look briefly at existing experimental data in Chapter 9 . 